\title{
Estimation of Integral Curves from High Angular Resolution Diffusion Imaging (HARDI) Data
}

\author{
Sakhanenko $\mathrm{L}^{1 *}$, DeLaura $\mathrm{M}^{2}$ and $\mathrm{Zhu} \mathrm{DC}^{3}$ \\ ${ }^{1}$ Department of Statistics and Probability, Michigan State University, USA \\ ${ }^{2}$ Department of Statistics and Probability, Michigan State University, USA \\ ${ }^{3}$ Department of Radiology, Michigan State University, USA
}

\begin{abstract}
We discuss the superiority of the high order tensor approach to HARDI, or High Angular Resolution Diffusion Tensor Imaging, over the probabilistic tractography approaches and the lower order tensor model (DTI, or Diffusion Tensor Imaging) discussed in. Probabilistic tractography methods, which utilize Monte Carlo sampling and bootstrap techniques, have a distinct disadvantage inherent in the need for assumed arbitrary priors in light of unknown circumstances (i.e., the obscure nature of the connective structure of the brain). In such situations, fewer model assumptions make more sense. This motivates our proposed method, which uses a semi-parametric high order tensor function with the ability to differentiate between multiple fiber directions. The ability to handle multiple or crossing fibers is the main advantage to the high order approach over DTI. Since it is known that such instances commonly occur in white matter, there is a clear need for a method which can effectively model this phenomenon. In this paper we discuss the advantages and disadvantages of the current works to highlight the reasons medics would improve analysis through our method. We also discuss the evaluation of performance on simulated and brain data.
\end{abstract}

\section{HARDI}

In the calculation of the model under the Bayesian approach, one must choose a "prior" distribution to combine with one's data, assumed to be the true distribution of the parameter of interest prior to the consideration of data. Unfortunately, these arbitrary priors are not robust and cannot perform well over a wide range of data. Situations will therefore inevitably arise in which our data disagrees with our prior belief.

Since the detailed anatomy of the brain is still uncharted in terms of connectivity due to high amount of noise, this approach runs a high risk for misplacement of curve estimates and so called "phantom images". The prior information for one region is not guaranteed to be applicable to any other region. Hence repeated sampling methods attempting to quantify uncertainty in the model may be used on an incorrect basis.

In light of this it makes sense for a completely data driven, or nonparametric, approach for the sake of robustness in obscure data one deals with in diffusion MRI analysis. To this end, we move towards a semi-parametric high order tensor model approach of the estimation of trajectories. Non-parametric or semi-parametric methods have inherited robustness to misspecification because they are completely data driven (or nearly completely) and fewer assumptions are made. The methods in [1-3] for DTI and HARDI models, both semiparametric approaches, have this advantage.

We provide a tracing of the fiber along with surrounding confidence ellipsoids so that scientists can better understand where the true fiber is located. Since the error in raw data measurements can result in a large error in curve estimates, we assess how much by following the curve with a surrounding confidence band. This method would enhance MRI analysis by helping to solidify confidence in correct interpretations of fiber locations by allowing the scientist to supplement information from original images with a region of confidence surrounding the regions of interest, as they indicate how reliable the location of the estimated fiber is (the tighter the confidence band, the more confident we are in that being the true location of the fiber). This is a one-step tracing process of the curve with its surrounding confidence ellipsoids, so that our method has the benefit of no iterative sampling. This makes it very advantageous in a computational sense. That is, we are carrying easily interpretable uncertainty through from the acquisition of the raw data with us to the curve tracing to obtain closed form estimates of tract uncertainty as opposed to the Bayesian approach, in which one needs to construct thousands of empirical curves in order to estimate the posterior curve distribution or perform repeated sampling methods for uncertainty quantification.

Another component of our method is the ability to perform hypothesis tests of whether two points in the brain are connected. Given some starting point, we can use properties of our curve estimators to construct a hypothesis test that the curve starting at the given initial point will pass through some other point of interest. One can test regions of interest or exhaustively to supplement analysis and improve interpretability of images.

While these advantages are pertinent to the approaches of both DTI and HARDI, high order tensor model approach can trace branching or intersecting curves. As mentioned before, this is highly preferred since it is known such branching and crossing phenomenons exist in the brain. In the paper [4] the high order tensor model is compared to the

Correspondence to: Sakhanenko L, Department of Statistics and Probability. Michigan State University, USA, E-mail: luda@stt.msu.edu

Received: February 16, 2018; Accepted: March 02, 2018; Published: March 05, 2018 
standard matrix model in all circumstances of fiber thickness, signalto-noise ratio and crossing fibers. It also yields tighter confidence ellipsoids by as much as a factor of 10 across all combinations of these parameters and by a factor of up to 1,000 for high signal-to-noise ratios. This is a huge advantage, since $\mathrm{C}, \mathrm{Y}$, or $\mathrm{X}$ shaped patterns (the ones that we observe the performance of our methods on) are commonly observed. Sequential combinations of these patterns can create any pattern one might expect to stumble across during analysis (or could imagine anatomically exists).

We first evaluated our method on simulated data. The data was simulated in a manner mimicking the information provided by the MRI image. We employed our method to assess its ability to trace common patterns. Since most tracings in the brain can be combined by these sequential $\mathrm{C}, \mathrm{Y}$, or $\mathrm{X}$ patterns, this makes sense to simulate such patterns and apply our method to assess how well we are able to trace the patterns. It is worth mentioning that the simulation of data for practical purposes is debatable due to the question of how to realistically simulate noise. Thus, another artificial dataset which simulates realistic fibers is the so-called tractometer [5]. A competition between different approaches revealed no single winner but showed how a fair comparison can be performed. Our approach performed excellent on this dataset using just the DTI model, see [6].

Our method performs well on brain data as well. In the paper [1] we summarized the analysis of the region of the white matter C-shaped area in the brain of the corpus callosum. The performance of the high order model yielded estimated curves consistent with the known anatomy of this region of the brain with small covariance. The method is currently being further used to explore the detailed anatomy of other regions of the brain and a paper is in preparation. For example, we applied our method to a diffusion-weighted imaging (DWI) dataset collected from a healthy adult brain on a GE 3T Signal HDx MR scanner (GE Healthcare, Waukesha, WI) with an 8-channel head coil.
The subject signed the consent form approved by the Michigan State University Institutional Review Board. DWI images were acquired with a dual spin-echo echo-planar imaging (EPI) sequence for 7 minutes and 45 seconds with the following parameters: 54 contiguous $2.4-\mathrm{mm}$ axial slices in an interleaved order, FOV $=22 \mathrm{~cm}$-by- $22 \mathrm{~cm}$, matrix size $=128$-by- 128 , number of excitations $(\mathrm{NEX})=1, \mathrm{TE}=86.6 \mathrm{~ms}, \mathrm{TR}=$ $15.5 \mathrm{~s}$, 25 diffusion-weighted volumes (one per gradient direction) with $\mathrm{b}=1000 \mathrm{~s} / \mathrm{mm} 2,4$ volumes with $\mathrm{b}=0$ and parallel imaging acceleration factor $=2$. Figure 1 summarizes our findings. Figure 1(a) shows the tracing of a fiber across a voxel at the central body of corpus callosum. The fiber is shown in red surrounded by the confidence band in black. Figure 1(b) shows the tracing of a fiber connecting the right and left lateral occipital cortical regions. The seed voxel is at the white matter near the right lateral occipital cortex. The fiber is shown in red surrounded by the confidence band in black. We use 95\% confidence level for both images [7].

\section{Conclusion}

Our high order tensor model approach is an improvement over the existing standard matrix model approach in terms of both tightness of confidence bands and performance for connectivity testing. It has the advantage of no arbitrary assumptions in the presence of highly unknown situations and does not require iterative sampling. This sensible and computationally inexpensive approach can greatly improve the analysis of brain connectivity by instilling more confidence in the interpretation of images. We make now a move toward an entirely non-parametric approach as a direction of future research work.

\section{Acknowledgment}

The authors report no conict of interest. Research was partially supported by the NSF grant DMS-1612867 and by AGEP supplement, NSF grant DMS- 1742881 .

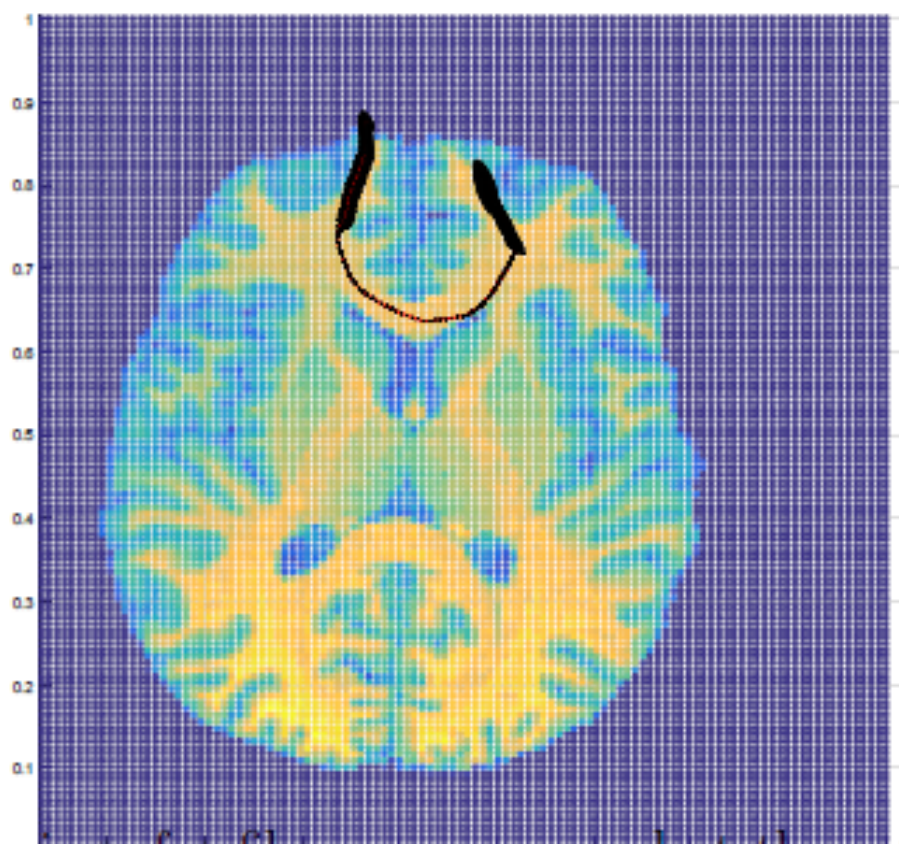

(a)

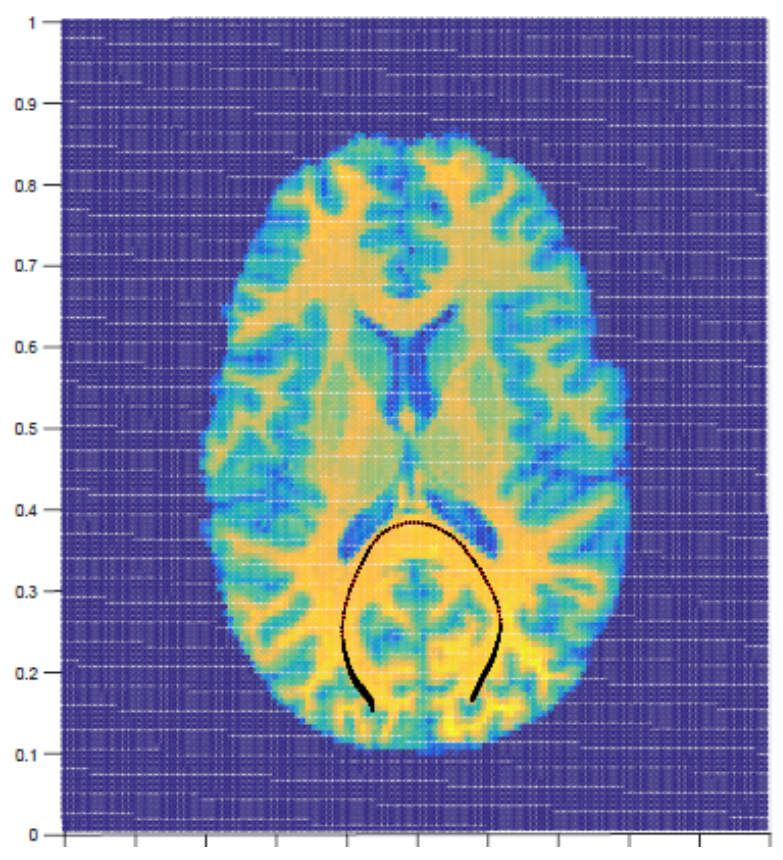

(b)

Figure 1. Real examples of images obtained via HARDI with estimated integral curves and confidence ellipsoids. A black $95 \%$ confidence band surrounds the estimated fiber. (a) Tracing of a fiber across a voxel at the anterior body of corpus callosum is in red. (b) Tracing of a fiber connecting the right and left lateral occipital cortical regions is in red. 


\section{References}

1. Carmichael O, Sakhanenko L (2015) Estimation of integral curves from high angular resolution diffusion imaging (HARDI) data. Linear Algebra and its Appl 473: 377-403. [Crossref]

2. Assemlal HE., Tschumperle D, Brun L, Siddiqi K (2011) Recent advances in diffusion MRI modeling: Angular and radial reconstruction. Med Image Anal 15: 369-396. [Crossref]

3. Carmichael O, Sakhanenko L (2016) Integral curves from noisy diffusion MRI data with closed-form uncertainty estimates. Statistical Inference for Stochastic Processes 19: 289-319.
4. Sakhanenko L, DeLaura M (2017) A comparison study of statistical tractography methodologies for Diffusion Tensor Imaging. International Journal of Statistics: Advances in Theory and Applications 1: 93-110.

5. Daducci A, Canales-Rodrguez E, Descoteaux M, Garyfallidis E, Gur Y, et al. (2014) Quantitative comparison of reconstruction methods for intra-voxel fiber recovery from diffusion MRI. 33: 384-399. [Crossref]

6. Sakhanenko L (2015) Using the Tractometer to assess performance of a new statistical tractography technique. Journal of Nature and Science 1: 1-12.

7. Sakhanenko L, DeLaura M (2017) A comparison study of statistical tractography methodologies for Diffusion Tensor Imaging. International Journal of Statistics: Advances in Theory and Applications 1: 93-110.

Copyright: (C2018 Sakhanenko L. This is an open-access article distributed under the terms of the Creative Commons Attribution License, which permits unrestricted use, distribution, and reproduction in any medium, provided the original author and source are credited. 